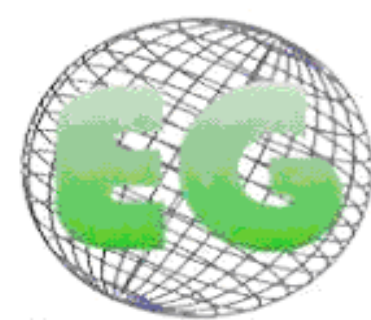

ISSN 1695-6141 $\mathrm{N}^{\circ} 22$
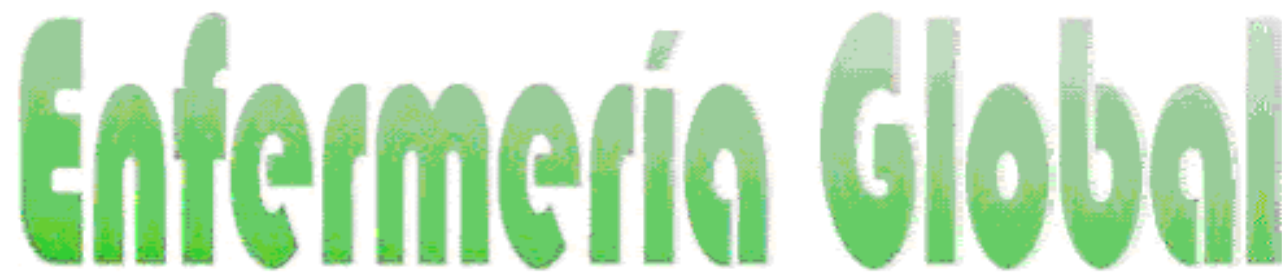

Revista electrónica trimestral de Enfermería

Abril 2011

\title{
ATENCIÓN AL ACCIDENTE CEREBRO VASCULAR INFANTIL: PERSPECTIVA DE LA NEUROLOGÍA PEDIÁTRICA
}

ATENDIMENTO AO ACIDENTE VASCULAR ENCEFÁLICO INFANTIL: PERSPECTIVA DE NEUROPEDIATRAS

\author{
*Liégio Matão, L. ${ }^{* *}$ Borges de Miranda, D. ${ }^{* * *}$ Faria Campos, PH. ${ }^{* * *}$ Pacheco, \\ LP. ${ }^{* * * *}$ Martins Pereira, $P$.
}

*Mestre em Enfermagem. Professora Assistente II do Departamento de Enfermagem da Pontifícia Universidade Católica de Goiás/PUC Goiás. **Enfermeiro. Especialista em Gestão de Sistemas e Serviços de Saúde. ${ }^{* *}$ Doutor em Psicologia. Professor Titular do Departamento de Psicologia da Pontifícia Universidade Católica de Goiás/PUC Goiás. ${ }^{* * * *}$ Enfermeira. Especialista em Terapia Intensiva. ${ }^{* * * * *}$ Enfermeira. Especialista em Auditoria em Serviços de Saúde. Goiás. Brasil.

Palabras clave: Accidente cerebral vascular; Cuidados médicos; Educación en Salud Palavras chave: Acidente cerebral vascular; Cuidados médicos; Educação em saúde .Keywords: Stroke; Medical Care; Health Education

\section{RESUMEN}

Objetivo: discutir en la perspectiva de la neurología pediátrica la atención del accidente cerebro vascular infantil en la ciudad de Goiania.

Método: el enfoque descriptivo y abordaje cualitativo. Entrevista abierta en profundidad con neuropediatras, siendo el material sometido a análisis de contenido. El estudio fue aprobado por el Comité de Ética en Investigación de la Pontificia Universidad Católica de Goiás (número de protocolo 95645-0339/06).

Resultados: los sujetos enfocaron, en sus narraciones, muchos más aspectos teóricos sobre la enfermedad en la infancia, los cuales aparecen de modo homogéneo y coincidente con la literatura especializada. El análisis reveló tres categorías, a saber: "Aspectos teóricos de accidente cerebro vascular en la infancia", "Atención hospitalaria (des)organizadao" y "Ausencia de educación en salud a la comunidad".

Conclusiones: el tratamiento del accidente cerebro vascular en Goiania pediátrica se presentó a la población de modo notoriamente distinto. Se puede inferir que la situación económica determina no sólo el acceso a los servicios que ofrece la última tecnología, sino también sobre la "calidad" de la atención, ya que no hay neuropediatras actuando en la red pública, por lo que el servicio es inferior al necesario. En el ámbito privado son mucho más fáciles, más rápidos y cualificados. 


\section{RESUMO}

Objetivo: discutir na perspectiva de neuropediatras, o atendimento do acidente vascular encefálico infantil na cidade de Goiânia.

Método: estudo descritivo e abordagem qualitativa. Foi realizada entrevista aberta em profundidade com neuropediatras, sendo o material submetido à análise de conteúdo. O estudo foi aprovado pelo Comitê de Ética em Pesquisa da Pontifícia Universidade Católica de Goiás (protocolo número 956450339/06).

Resultados: os sujeitos enfocaram, em suas narrativas, muito mais aspectos teóricos acerca do agravo na infância, os quais aparecem de modo homogêneo e coincidente com a literatura especializada. Emergiram três categorias de análise, quais sejam: "Aspectos teóricos do acidente vascular encefálico na infância", "Atendimento hospitalar (des)organizado" e "Ausência de educação em saúde à comunidade".

Conclusões: o atendimento do acidente vascular encefálico pediátrico em Goiânia se apresenta à população de modo flagrantemente distinto. Pode-se inferir que a condição econômica dita não só acesso a serviço que dispõe de tecnologia de ponta, mas também acerca da "qualidade" da assistência, uma vez que não existem neuropediatras atuando na rede pública, portanto, o atendimento está aquém do necessário. Na esfera privada são expressivamente mais fáceis, rápidos e qualificados.

\section{ABSTRACT}

Objective: to discuss the prospect of pediatric neurology, care of stroke in the infant city of Goiania.

Method: descriptive and qualitative approach. An interview with neuropediatricians open at depth, the material was subjected to content analysis. The study was approved by the Ethics in Research of the Catholic University of Goiás (protocol number 95645-0339/06).

Results: the subjects focused on their narratives, much more theoretical aspects about the disease in childhood, which appear seamlessly and coincided with the literature. Analysis revealed three categories, namely: "Theoretical aspects of atroke in childhood", "Care hospital (dis)organized" and "Absence of the community health education".

Conclusions: the treatment of pediatric stroke in Goiania is presented to the public so blatantly different. One can infer that the economic condition dictates not only access to service that offers the latest technology, but also about the "quality" of care, since there are no neuropediatricians acting in public, so the attendance is lower than necessary. In the private sphere are significantly easier, faster and skilled.

\section{INTRODUCCIÓN}

Niño es sinónimo de salud, alegría, diversión y educación. En Brasil, el Estatuto del Niño y del Adolescente establece que todos los niños y los adolescentes gozan de los derechos fundamentales inherentes a la persona humana, garantizando a todos las oportunidades e instalaciones con el fin de proporcionarles el bienestar físico, mental, condiciones morales, espirituales y sociales libertad y dignidad. Es deber de la familia, comunidad, gobierno y sociedad en general garantizar, con absoluta prioridad, la aplicación efectiva de los derechos a la vida, la salud, nutrición, educación, deporte, ocio, formación profesional, la cultura, la dignidad, el respeto, la libertad, la familia y la Comunidad 
A pesar de estos derechos garantizados por la ley, hay niños que no los disfrutan plenamente, debido a múltiples factores, especialmente los efectos negativos para la salud. Entre las enfermedades que pueden afectar a los niños aparecen tanto enfermedades de rápida recuperación como patologías que causan secuelas temporales o irreversibles, como accidente cerebro vascular (ACV), que en algunos casos, dificulta o impide que el niño hable, camine, estudie, se alimente y, finalmente, se desarrolle plenamente.

Un ACV ocurre con menos frecuencia en comparación con las patologías posibles que se dan en ese período de la vida, pero cuando se instala, el impacto puede ser muy grave. Debido a la reciente introducción de métodos de diagnóstico más específicos, se lograron avances, aun así, hay casos en que el establecimiento de la etiología no se hace.

ACV es una enfermedad caracterizada por la aparición repentina de déficit neurológico (disminución de la función), con reflejo focal en el sistema nervioso central (SNC) como consecuencia de la perturbación de la circulación cerebral. Esta patología se puede clasificar en dos tipos: isquémico y hemorrágico. El isquémico consiste en la oclusión de un vaso sanguíneo que interrumpe el flujo de sangre a una región específica del cerebro, lo que interfiere con las funciones neurológicas dependientes de esa área afectada. El sangrado se produce al romper la pared de una arteria cerebral, por lo tanto se produce salida de la sangre, aumento de la presión intracraneal y edema cerebral. ${ }^{2}$

La enfermedad cerebro vascular es muy común en los países industrializados. Según la Organización Mundial de la Salud (OMS) el derrame cerebral afecta a 15 millones de personas al año y es la tercera causa de muerte natural en el mundo. De ellos, cinco millones mueren y otros cinco millones sufren daño neurológico permanente. Aproximadamente uno de cada cuatro hombres y una de cada cinco mujeres puede sufrir un ACV hasta 85 años de edad. En Brasil, 129.172 personas murieron por accidente cerebrovascular en el año 2002; se estima que las enfermedades cardiovasculares están entre las principales causas de muerte en las grandes ciudades ${ }^{3}$

En el caso de la infancia, el ACV es multifactorial. Aparecen como las más importantes enfermedades hematológicas (la anemia de células falciformes, policitemia, enfermedad Púrpura autoinmune del recién nacido), las enfermedades autoinmunes, malformaciones arteriovenosas, aneurismas, enfermedades del riñón, displasia fibromuscular, mitocondriopatía, SIDA, trastornos vasculares, enfermedades del corazón, deshidratación, shock séptico y el cáncer. Causas relacionadas con la coagulación hereditaria, con la participación mutaciones en el ADN genómico, y que afecta en particular el factor $\mathrm{V}$ y factor II, también son causas importantes, pero que todavía están en estudio. A pesar de numerosas causas, muchos casos de ACV infantil no han aclarado su etiología. ${ }^{4-6}$

Entre los tipos de ACV en la infancia, el isquémico es el más frecuente. Afecta fundamentalmente a la zona irrigada por la arteria cerebral media, siendo la izquierda su mayor parte lesionada. Esto a su vez se divide en embólicos, con embolia cardíaca, séptico, gaseoso, graso y la trombosis, con la posibilidad de trombosis arterial y senos venosos. ${ }^{6-8}$

Según las estadísticas realizadas en los países desarrollados, el derrame cerebral infantil afecta aproximadamente 15/100.000 habitantes y año. En Brasil, no existen estadísticas, pero se estima alrededor de 18/100.000 habitantes y año. Estudios en el vivero de alto riesgo del Centro de Atención Integral a la Salud de la Mujer en la UNICAMP, se identificaron 25 casos de esta lesión en los recién nacidos cada 10000 nacimientos, aproximadamente ocho casos por año. ${ }^{9}$ 
En Brasil, el estudio sobre los accidentes cerebrovasculares en los niños se inició en los años 90. En ese momento, la gente no creía en la posibilidad de que ocurriesen casos de la enfermedad en niños, pensamiento que dura hasta hoy. Con el establecimiento de los recursos técnicos de exploración no invasiva, el diagnóstico se hizo más confiable, porque antes eran puramente clínicos. ${ }^{9}$

Ante lo expuesto, se analiza en este estudio, desde la perspectiva de la neurología pediátrica, el cuidado de niños con accidente cerebro vascular en la ciudad de Goiania.

\section{MÉTODO}

Se trata de una investigación de campo, de un estudio exploratorio, cualitativo y descriptivo. Esta alternativa permite explorar y describir los fenómenos dirigidos a la percepción, la intuición y la subjetividad del sujeto, centrándose en un universo de significados, motivos, aspiraciones, creencias, valores y actitudes. ${ }^{10}$

El estudio se inició después de la aprobación por el Comité de Ética en la Investigación de la Universidad Católica de Goiás (número de protocolo 95645-0339/06). Participaron como sujetos neuropediatras, sin importar la titulación obtenida tras la referida especialidad y el tiempo de formación académica, con trabajo en instituciones de salud en la ciudad de Goiania.

La recolección de datos se realizó mediante dos instrumentos: uno se refiere a la realización de la forma (registros socioeconómico y atención especializada llevada a cabo en el sector privado y público) y el otro, entrevistas abiertas en profundidad, que se inició con la pregunta: "¿Puede relatarnos cómo ha sido el cuidado de los niños con ACV en los servicios públicos y privados en Goiânia, y conoce la realización de trabajos para alertar a la población sobre la enfermedad en la infancia? El criterio de saturación fue el elegido para el cierre de la recogida de datos.

La mayoría de las entrevistas fueron grabadas con el consentimiento de los sujetos, quienes validaron después la transcripción; uno de ellos no permitió la grabación, por lo que se hizo un registro manual.

Con el fin de mantener el anonimato, los participantes fueron rebautizados con los nombres de las estrellas más brillantes del firmamento. Los preceptos de la Resolución 196/96 ${ }^{11}$ se cumplieron. Se utilizó el análisis de contenido para establecer los temas. ${ }^{12}$

\section{RESULTADOS}

En Goiania, hay diez neuropediatras, que se ofrecieron para participar en la investigación como sujetos de investigación, ya sea por contacto personal o por teléfono. De éstos, solo cinco consintieron formar parte del estudió. Las justificaciones para la no participación fueron: no trabajar con un ACV en la infancia y sí con la psiquiatría infantil y/o la falta de tiempo.

\section{Caracterización del grupo}

Los sujetos de estudio presentaron un promedio de 18,4 años de formación académica, mínimo de nueve y un máximo de 39 años. Todos los residentes en neurología y pediatría, la mayoría con evidencia de especialización. En cuanto al desarrollo profesional, todo el mundo dice que lo hace a través de la participación en conferencias y lectura de revistas 
profesionales, algunos también llevan a cabo cursos y buscan nuevos conocimientos en Internet; entre los participantes uno se integra en un grupo de estudio.

Todos los neuropediatras que concedieron entrevista trabajan en las instituciones privadas de referencia en la atención pediátrica y/o neurológica. En el grupo hay unanimidad acerca de las condiciones en las instituciones privadas para atender adecuadamente a estos niños, ya sea para el diagnóstico y tratamiento en la fase aguda, ya sea para la rehabilitación de las secuelas, tanto en lo referente al número de personal como también material y equipo especializado. Sobre esta capacidad en el sector público no existe un consenso y las opiniones van desde la incapacidad para emitir juicio hasta la discrepancia entre la disponibilidad de equipo o no, lo que de alguna manera hace pensar en la falta de preparación para la investigación de la patología en este ámbito. En lo que respecta al número de niños víctimas de accidente cerebro vascular, en Goiania, la media reportada fue de dos casos por año.

\section{Análisis fenomenológico}

\section{- Aspectos teóricos de accidente cerebro vascular en la infancia}

La narrativa de los sujetos fue iniciada por enfoque de contenido plenamente teórico acerca del agravio en los niños. Esta categoría de análisis fue dividida en tres subcategorías denominadas de la siguiente manera: "Génesis", "El Diagnóstico" y "Terapia".

\section{- El Génesis}

Entre las muchas causas que pueden atribuirse a la ocurrencia de ACV en la infancia se centraban unánimemente entre los encuestados, aquellos identificados como base de las cardiopatías congénitas, enfermedades hematológicas y vasculitis, como se demuestra en el discurso:

[...] Son varios los factores que causan el ACV infantil, principalmente las dolencias hematológicas (anemia), cardiopatías, dolencias autoinmunes y asociación de síndrome de Down y enfermedad de Moya-Moya [...] (Sirius).

[...] ¿Lo que causa el ACV en la infancia? La gente determina tres aspectos, los problemas cardiológicos..., dolencias hematológicas... y enfermedades raras como anemia falciforme que en el ACV no es tan raro [...] (Antares).

La enfermedad cerebro vascular se debe principalmente a la insuficiencia de la arteria cerebral media, con perjuicio de las funciones previstas en la parte del cerebro afectada, que fue detallado por uno de los participantes de la siguiente manera:

"La arteria cerebral media irriga parte de los lóbulos frontal, parietal y temporal, que son responsables de las funciones sensitivas y motoras.

La mayoría de los derrames en razón del comportamiento de esa arteria o sus ramas, ocurre, principalmente por isquemia y trombosis, de esa arteria, o sus ramas". (Sirius)

Sin embargo, no siempre es posible determinar la causa de ACV en los niños. Entre los temas hay consenso en este aspecto con algunas divergencias en el porcentaje de casos: 
"En apenas $20 \%$ de los casos se consigue identificar la dolencia de base y otro $80 \%$ son iatrogénicos, no identifica la causa" (Estrela Dalva).

"Cerca de 55\% de los casos tienen su etiología definida y $45 \%$ no tiene causa establecida, lo que favorece los casos de recidiva". (Sirius)

La enfermedad cerebro vascular en la infancia se desencadena por diversos factores, ya que hay casos donde la enfermedad subyacente no se conoce, por lo que es necesario un estudio detallado y el diagnóstico precoz.

\section{- Diagnóstico}

En la búsqueda para determinar el diagnóstico y la etiología se recoge la historia clínica y pruebas de laboratorio y estudios de imagen. A pesar de manifestarse de forma insidiosa, una cuidada colecta de datos sobre la historia clínica es fundamental:

[...] En la mayoría de los casos la madre solo percibe alguna alteración cuando, por ejemplo, la criatura pierde la fuerza muscular y cae [...]. (Sirius)

[...] En medicina hay una cosa muy importante que se llama historia, esta tiene que estar bien hecha y es lo más difícil de la medicina, hoy, todos quieren hacer exámenes [...]. (Polaris)

Su identificación no es tarea fácil, de ahí el interés en los estudios de neuroimagen. En los últimos años, entre las pruebas, la resonancia magnética y la tomografía computarizada son los procedimientos de ayuda en diagnóstico más accesibles y seguros, por lo tanto, se utilizan como primera elección en estos casos:

"Son realizados con urgencia tomografía computadorizada y resonancia magnética, si es necesario, en el mismo día son realizados exámenes complementarios". (Estrella Dalva)

El grupo se posiciona en contra del uso de exámenes como la primera opción para llegar a un diagnóstico:

"El examen es complementario, hacer examen de forma innecesaria es expuesto para el paciente y cuesta caro". (Polaris)

Determinar la causa del accidente cerebrovascular en los niños es esencial para establecer el adecuado abordaje terapéutico, basado principalmente en la atención de las complicaciones cardíacas y respiratorias. El diagnóstico preciso asegura una mejor preparación de estrategias para la rehabilitación de niños víctimas de esta lesión.

- $\underline{\text { Terapéutica }}$

Una vez diagnosticada la enfermedad cerebro vascular, el tratamiento debe establecerse inmediatamente para frenar la creación de secuelas y permitir la rehabilitación con resultados más satisfactorios. 
"El tratamiento debe ser iniciado lo más rápidamente posible, para impedir el aumento de la lesión". (Sirius)

En el tratamiento del ACV infantil en la fase aguda, existe un consenso entre los grupos sobre estabilización hemodinámica y soporte ventilatorio.

[...] Ahora, desde el punto de vista del tratamiento del ACV en la infancia, e episodio agudo no tiene tratamiento, básicamente es estabilizar a la criatura, colocarla en una UTI, estabilizar hemodinámicamente al niño $y$, si estuviera grave, colocar en soporte ventilatorio... y principalmente investigar la causa del ACV [...]. (Antares)

[...] Lo más importante es estabilizar, ventilar y asistir al enfermo, vigilar de verdad [...]. (Polaris)

El uso de medicación en la fase aguda de la enfermedad es diferente entre los sujetos, porque no hay un protocolo de servicio como en el accidente cerebrovascular en los adultos:

[...] Dependiendo del caso entra con medicamento como antiagregante plaquetario y vasodilatador cerebral [...]. (Estrella Dalva)

[...] Para el adulto ya existen protocolos con uso de fibrinolíticos y es usada hasta tres horas después del episodio de ACV. ¿Por qué esto no es usado en la infancia? Porque no existen trabajos asegurando la eficacia y sobre todo la seguridad de ese tipo de tratamiento en la infancia [...]. (Antares)

Pasada esta fase, en general, quedan consecuencias o secuelas, cuyo proceso de rehabilitación debe incluir el equipo multidisciplinar

"Dependiendo del área afectada es solicitada fonoaudiología, fisioterapeuta y terapia ocupacional, el paciente es encaminado para rehabilitación de acuerdo con la sintomatología". (Estrella Dalva)

De acuerdo con los entrevistados, el niño tiene un grado de recuperación de la función neurológica mayor que el del adulto, de ahí la importancia de iniciar rápidamente el tratamiento:

"Como el cerebro de los niños tiene mayor capacidad de
neuroplasticidad, las áreas no afectadas por la lesión intentan
asumir las funciones de las áreas afectadas, la rehabilitación
supone un grado de mejora más elevado que en el adulto". (Sirius)

EI ACV puede llegar a grandes áreas del territorio del cerebro, provocando consecuencias graves e irreversibles, por lo que la rehabilitación en curso las necesidades, como lo demuestra el discurso de un entrevistado: 
"La rehabilitación del niño debe ser para el resto de su vida, dividiéndose en dos fases, la primera consiste en alcanzar el nivel máximo de mejora, y la segunda consiste en asegurar la mejora alcanzada”. (Sirius)

La enfermedad cerebro vascular es un enfermedad de etiología diversa, no siempre identificada, cuyo diagnóstico se establece por laboratorio clínico y de neuroimagen. Esta lesión incita a numerosas secuelas, por lo que el tratamiento debe iniciarse de forma precoz para fomentar una mayor rehabilitación del niño.

Sin embargo, para que el niño víctima de ACV sea atendido adecuadamente, y reducir al mínimo las consecuencias, es necesario que las instituciones públicas y privadas se preparen tanto con profesionales como con equipos especializados para el diagnóstico, tratamiento y rehabilitación.

\section{- Atención hospitalaria (DES) Organizado}

Según el grupo, la asistencia de la enfermedad en la infancia es comparativamente diferente en las instituciones de red privada y red pública. Las organizaciones privadas tienen equipo profesional y especializado, lo que favorece el diagnóstico precoz.

[...] En la red privada el niño es encaminado al pediatra, especialista o por medio de parecer, cuando el niño está internado, al neuropediatra [...]. (Estrella Dalva)

[...] Yo trabajo en un hospital de referencia e intentamos hacer lo mejor, mas no es común ver un $A C V$ en niños... aquí tienen una manera de conducir cuando tienen un diagnóstico de $A C V$, tienen una rutina de investigación [...]. (Polaris)

En contraste, las escuelas públicas tienen una capacidad limitada para el diagnóstico precoz.

[...] En la red pública la mayor dificultad es establecer el diagnóstico precoz, siendo preciso, muchas veces, internar al niño sin necesidad, exponiéndolo al riesgo de infección hospitalaria, para realizar exámenes complementarios como tomografía y resonancia, y existe también una larga lista de espera por el "chequim". Quedo frustrada con a burocracia del SUS, no se consiguen exámenes complementarios [...] El SUS es muy bonito sobe el papel para concurso, mas no funciona [...], en el SUS es preciso esperar seis meses para la primera consulta de rehabilitación...]. (Estrella Dalva)

Durante las entrevistas, dos sujetos, inicialmente, manifestaron no tener una diferencia en el tratamiento del ACV entre los niños de instituciones públicas y privadas, sin embargo, e después enfocan la temática de forma contraria. Según ellos, la red privada tiene un equipo multiprofesional y equipo especializado para dar soporte adecuado a los niños víctimas de ACV. Mientras que la red pública es limitada en términos de diagnóstico precoz de la enfermedad, pero:

"A nivel ambulatorio para el acompañamiento de secuelas tal vez tenga más estructura, mas para la atención de urgencia es más difícil. En la institución particular es mejor". (Mira) 
Independientemente de si se es miembro de la red pública o privada, el hecho es que ante cualquier señal y síntoma sugestivos de esa enfermedad, a la institución de salud debe acudirse inmediatamente. Por lo tanto, es necesario aclarar a la comunidad lega sobre la posibilidad de que ocurra la enfermedad en la niñez.

\section{- La ausencia de Educación para la Salud de la Comunidad}

La divulgación de la enfermedad cerebro vascular en la población lega es punto importante en la identificación de signos y síntomas tempranos y, en consecuencia, la demanda de servicio rápido. Sin embargo, la no existencia de ningún trabajo para informar a la población sobre el ACV en la infancia se percibe unánime entre los entrevistados.

"No existe trabajo preventivo para la población infantil como existe para pacientes adultos". (Sirius)

"No tengo conocimiento de trabajos para alertar a la población sobre la enfermedad en la infancia". (Mira)

Debido a que el ACV en los adultos tiene altas tasas de incidencia, se ofrece al público información sobre la enfermedad, la identificación y persecución de un servicio rápido, tal como se especifica a continuación:

"Para adultos existe una serie de trabajos, inclusive el Día Nacional de Combate al ACV, que es coordinado por la Academia Brasileira de Neurología, en todas las capitales, inclusive la nuestra”. (Sirius).

Uno de los encuestados cree que la falta de trabajos con los legos, sobre todo enfocados en la ocurrencia en la infancia, se produce debido a la rareza de la enfermedad; con todo, hay trabajos para la comunidad científica, como demuestra el siguiente discurso:

[...] Existen innumerables trabajos para la población médica, son trabajos científicos. [...] No hay trabajos epidemiológicos ni datos epidemiológicos lo que, hasta hoy, limita un trabajo con la población [...] (Antares)

La falta de educación sanitaria a la comunidad en relación con el ACV infantil, deriva también de la falta de capital y disponibilidad de tiempo según lo especificado en el discurso a continuación:

"No existen trabajos para alertar a la población, porque para eso se precisa dinero y tiempo, y nosotros no tenemos tiempo y mucho menos dinero". (Polaris)

De acuerdo con lo anterior, la población lega, prácticamente, no tiene conocimiento de esta patología, resultando un retraso en el diagnóstico por la demora en buscar atención, lo que aumenta el riesgo de secuelas.

\section{DISCUSIÓN}

La experiencia de la neurología pediátrica en relación con el tiempo de aprendizaje del alumno, les permite enviar sus experiencias y opiniones de una manera segura, ya que dicha asistencia está a cargo de ellos. 
Inicialmente, dos hechos en la caracterización de los participantes llamaron la atención: el pequeño número de especialistas en el tema (de neuropediatras), así como la falta de actuación de la categoría en la red pública. Hay en Goiania diez neuropediatras, de los cuales cuatro operan en el área de psiquiatría infantil. Posiblemente, este reducido número de especialistas se debe a la necesidad de hacer dos residencias, pediatría y neurología, para obtener el título de neuropediatra, lo que requiere inversión y tiempo disponible.

La falta de neuropediatras en la red pública se justifica por el hecho de que la especialidad es costosa para la formación. En las instituciones públicas la cantidad pagada por cada procedimiento que se realiza es mucho menor que en el sector privado y, además, está el proceso de desguace de los hospitales públicos en términos de infraestructura, equipamiento y recursos humanos, y una incapacidad para incorporar, con agilidad las nuevas tecnologías en el ámbito estrictamente médico o de gestión. Los recursos financieros disponibles no siempre son suficientes para comprar el equipo con la última tecnología, o su mantenimiento adecuado, motivo por el que los profesionales cualificados y formados difícilmente se someten a esas condiciones. También contribuye a la no permanencia, los salarios bajos y la exigencia en el cumplimiento de la carga de trabajo impuesta. Todos estos aspectos sumados llevan a la falta de motivación de los profesionales en general a trabajar en esas instituciones.

En cambio, en la red privada, no existe una mayor disponibilidad de acceso a los exámenes de neuroimagen y de laboratorio, lo que favorecer el diagnóstico precoz, por lo tanto, las secuelas menos graves y derivación a rehabilitación con una mayor probabilidad de recuperación, toda vez que el aspecto financiero, en cierto sentido, no es impedimento para el acceso a la tecnología y equipo especializado.

Aunque pocos médicos trabajan con niños ACV, en la práctica hay casos, que recientemente provocaron la atención de los profesionales en el tema. Esta movilización se produce en una etapa en que los medios de diagnóstico se hicieron más fáciles con el avance de la tecnología de neuroimagen, como la tomografía computarizada y la resonancia magnética. La llegada de los equipos de neuroimagen sofisticada y recursos de laboratorio en los líquidos orgánicos facilitó la identificación de los tiempos del ACV infantil, de acuerdo a la literatura. ${ }^{6}$ Sin embargo, en instituciones públicas, los recursos tecnológicos son limitados, ya que no se adquirieron o están dañados, lo que dificulta la investigación de diagnóstico y la prestación de asistencia ineficaz para los niños víctimas de esta lesión.

En Brasil, los primeros estudios científicos sobre el ACV en los niños se iniciaron en 1990, cuando la neuróloga María Valeriana Leme-Moura Ribeiro creó el grupo de estudio sobre el ACV infantil. Desde entonces, otros estudios se prepararon, sin embargo de manera tímida, porque es una enfermedad poco conocida por la comunidad científica y desconocida por la lega, y poco comprendida y aceptada entre la población. ${ }^{9}$

Por lo tanto, es necesaria la búsqueda constante de esos conocimientos nuevos, que hoy se puede lograr por diversos medios eficaces. Entre el grupo, ello se da mediante la participación en conferencias y lectura de revistas profesionales, cursos, búsquedas en Internet y la formación de grupos de estudio, estando esos recursos disponibles para adquirir y actualizar el conocimiento más actual y puntero..

Se centraron en las narrativas los aspectos más teóricos de la enfermedad en la infancia, que aparecen de modo homogéneo y coincidente con la literatura especializada. Los aspectos relacionados con la atención diaria, incluidos los factores subjetivos como las 
emociones y pensamientos, casi no aparecen en el discurso de los participantes. Se podría pensar que la baja incidencia de los casos atendidos contribuye a este resultado. Probablemente, esto sucede también porque es más conveniente para los profesionales demostrar el dominio objetivo que poseen de la zona de actividad, lo que favorece la vanidad y el poder. Demostrar sentimientos y percepciones puede ser entendido como vulnerabilidad y preguntas, respectivamente.

En los países desarrollados, las estadísticas indican que el ACV infantil afecta a cerca de 15 niños por cada 100000 habitantes por año. En Brasil, no existen estadísticas oficiales, pero se estima alrededor del 18 por 100.000 habitantes por año. ${ }^{9}$ Según los sujetos, la enfermedad cerebro vascular en los niños es a menudo considerada una enfermedad rara, con un promedio de dos casos por año en Goiania. La ausencia de datos epidemiológicos, es el resultado de la suma de una serie de factores, entre ellos la dificultad para establecer el diagnóstico y el registro de los casos.

El trastorno cerebro vascular puede ser isquémico o hemorrágico, siendo el primero que más afecta a los niños. De hecho, una posible explicación es que el padecer una hemorragia cerebral determina una mayor mortalidad en la fase aguda, incluso antes de la realización del diagnóstico, mientras que el isquémico presenta mayores posibilidades de supervivencia, sin embargo, acarrea un gran número de secuelas. En cuanto a la región más afectada, es irrigada por la arteria cerebral media, específicamente la parte de los lóbulos frontal, parietal y temporal, que son responsables de las funciones motoras y sensoriales. Las principales causas se producen debido a la participación isquémica o trombótica de la arteria cerebral media izquierda, lo que refleja un déficit motor caracterizado por hemiparesia derecha, trastornos visuales, del comportamiento y aprendizaje, y la epilepsia. ${ }^{6,13}$

Tanto la literatura como los neuropediatras participantes en el estudio, evidencian las cardiopatías, vasculopatías y las enfermedades hematológicas, especialmente la enfermedad de células falciformes como las principales causas de ACV en la infancia. Sin embargo, las enfermedades infecciosas como el SIDA, la varicela y la meningitis son enfermedades que no se pueden descartar como un factor de riesgo para el ACV infantil, ya que muchos casos se producen durante el proceso infeccioso. A pesar de una cuidadosa investigación, algunas de las causas permanecen sin identificar. ${ }^{13}$

Es de notar la dificultad en el diagnóstico de la enfermedad cerebro vascular en la infancia, especialmente en recién nacidos y lactantes en los que, con frecuencia, la semiología es poco expresiva. Según lo citado en la entrevista y en la literatura, existe la posibilidad de que algunos niños hayan sido atendidos varias horas después de la instalación de la agresión, puesto que los signos y síntomas iniciales no fueron recuperados hasta el momento de los síntomas epilépticos y trastornos motores.

La demora en la autorización para realizar las pruebas costosas como la tomografía computarizada y la resonancia magnética es otro error identificado en la red pública. Hay una gran demanda de estas pruebas porque no es sólo el derrame cerebral el que necesita de los mismos para un diagnóstico concluyente, junto con esto, no están todos los establecimientos de salud que realizan los exámenes de alto costo por el SUS.

Los organismos públicos de Goiânia, así como muchas otras ciudades en el país, no tienen neuropediatras en su cuerpo clínico. Cuando el cuidado infantil es realizado por profesionales sin formación específica, la detección de signos y síntomas compatibles con un accidente cerebro vascular puede verse comprometida porque a menudo son pasados por alto $o$ no se valoran. Los pediatras sin formación en neurología no desarrollan los 
conocimientos técnicos especializados para detectar los leves signos de deterioro del motor en un hemisferio. ${ }^{6}$

El Ley Federal Brasileña $N \stackrel{0}{0}$ 8.080/90, que informa a la Constitución Federal de 1988 reafirma la salud como un derecho humano fundamental y un deber del Estado crear las condiciones esenciales para su pleno disfrute, asegurando el acceso universal e igualitario a acciones y servicios para su promoción, protección y recuperación. Sin embargo, la comunidad ha disfrutado de este derecho en virtud de la función pública. En la práctica, lo que ocurre en el caso de la asistencia a los niños víctimas de accidente cerebro vascular, son asistencias precarias debido a la falta de profesionales capacitados, equipamientos disponibles y la demora en la resolución de problemas. $^{14}$

Existe una superposición entre la literatura y los resultados obtenidos aquí con respecto a la importancia del diagnóstico precoz. Desde el diagnóstico preciso es posible diseñar estrategias para la atención terapéutica, estableciendo, a continuación, medidas de soporte hemodinámico y respiratorio, ya que la enfermedad cerebrovascular en niños no tiene el protocolo del tratamiento durante la fase aguda, ya que existe en el ACV en adultos que es promovida por la Sociedad Brasileña de las enfermedades. Luego de estabilizar al niño en la fase aguda e identificar las consecuencias, deben ser remitidos a la rehabilitación multidisciplinar con el seguimiento, de acuerdo a los cambios causados por la enfermedad, así como entre los adultos afectados. ${ }^{15}$

La educación sanitaria a la comunidad es el punto fundamental que contribuye a la concienciación de la sociedad en la búsqueda de atención de las enfermedades en general, de manera rápida. En relación con la atención en el accidente cerebro vascular, el factor tiempo está directamente relacionado con la intensidad de secuelas, específicamente cuanto mayor es el intervalo entre el inicio del evento y el inicio del tratamiento mayor es la gravedad de las mismas. El conocimiento puede alertar a la población acerca de los signos y síntomas de la lesión inicial, y su localización, busque un servicio rápido, por lo que las posibilidades de supervivencia de los niños son mayores, así como la mejora de su calidad de vida

Sin embargo, en Brasil no existen programas de educación sanitaria en este contexto, debido a factores no bien establecidos, reconocidos o revelados. La comprensión de los participantes, se puede atribuir a la falta de tiempo, falta de capital, un pequeño número de casos y sobre todo por ser un trastorno cuya actuación profesional en mayor escala es reciente.

El ACV en los niños no está muy extendido, hasta incluso en el ámbito académico y profesional, porque es una patología de preocupación e investigación reciente. Para el ACV en adultos hay información y sensibilización para la población como el Día Nacional de Lucha contra la ACV, coordinado por la Academia Brasileña de Neurología, lo que no sucede para los niños.

Aunque es poco frecuente en la infancia, la enfermedad cerebro vascular puede tener un impacto considerable sobre la vida del niño. Puede verse afectado en el rendimiento cognitivo y / o en el motor, incluso anunciar, en algunos casos, una enfermedad sistémica subyacente. Un niño afectado por ACV tiene su niñez saludable interrumpida, ya que cuando el ataque no es letal, deja marcas para toda su vida, y sin duda este niño enfrentará situaciones de exposición, y hasta prejuicios, dependiendo de la ayuda de los responsables por un período más largo, si no para toda la vida. 


\section{CONCLUSIONES}

El ACV en la infancia es una enfermedad que causa sorpresa a toda la sociedad, desde la población científica a la lega, sobre todo a esta última. También los estudios especializados centrados en la población infantil son recientes, lo que puede ser verificado por la dificultad de encontrar programas científicos y educativos en salud comunitaria a este respecto

El desafío en la lucha contra la enfermedad cerebrovascular en los niños es para establecer el diagnóstico y tratamiento tempranos. Por lo tanto, es necesario que las instituciones de salud, públicas y privadas, estén preparadas tanto en recursos humanos y en equipamiento para que los niños víctimas de ACV puedan recibir una atención adecuada y rápida, para minimizar las consecuencias y permitir una rehabilitación satisfactoria. Por esta razón, cada equipo debe ser consciente de la importancia de adquirir conocimientos técnicos en beneficio del niño pues todos los esfuerzos en la aplicación de estas habilidades deben tener prioridad en la promoción, protección y recuperación de la salud. Vale la pena subrayar la importancia de un equipo multi e interdisciplinario en todos los niveles de atención, con especial atención al proceso de educación para la salud.

Posiblemente, la condición que implica la atención pediátrica de ACV en Goiania, que se presenta a la población de manera evidentemente distinta de la ofrecida a la población adulta, es la realidad de la mayoría de los municipios. Otro aspecto a considerar es la posibilidad de inferir que la situación económica en este caso determina la calidad de la atención: los profesionales cualificados (neurología pediátrica) y la tecnología no están disponibles en la esfera pública sino en la privada, cuya asistencia es mucho más fácil, más rápida y cualificada.

Establecer la descripción de los elementos importantes en relación con este tema, es esencial para disponer de un proceso de reflexión entre los profesionales y directivos, principalmente de las instituciones públicas.

\section{REFERENCIAS}

1. Casa Civil (Brasil). Lei no 8.069, de 13 de julho de 1990. Dispõe sobre o Estatuto da Criança e do Adolescente e dá outras providências. Brasília: Diário Oficial da União, 1990 Jul 13. [acesso em 2010 Jan 20]. Disponível em: < http://www.planalto.gov.br/ccivil/LEIS/L8069.htm >

2. Aspesi NV, Gobbato PL. ABC da Saúde [homepage na Internet]. [atualizada em 2010 maio 08; acesso em 2010 maio 18]. Acidente vascular cerebral; [aproximadamente 4 telas]. Disponível em: <http://www.abcdasaude.com.br/artigo.php?6>.

3. Costa LF, Souza G, Pereira AH. Estenose da carótida extracraneana: análise dos fatores de risco e das indicações cirúrgicas. Rev angiol cir vasc. 2000;9(4):140-5.

4. Noce TR, Fabio SRC, Siqueira Neto JI, Santos AC, Funayama CAR. Cerebral infarct in children aged zero to fifteen years. Arq Neuro-psiquiatr. 2004;62(1):38-43.

5. Montenegro MA, Guerreiro MM, Scotoni AE, Tresoldi AT, Moura-Ribeiro MVL. Doença cerebrovascular na infância: manifestações epilépticas. Arq. Neuro-psiquiatr. );57(3A):587-593.

6. Moura-Ribeiro MVL, Ferreira LS, Montenegro MA, Valle-Cavalcante M, Piovesana AMSG, Scotini $A E$, et al. Doença cerebrovascular na infância: aspectos clínicos em 42 casos. Arq. Neuro-psiquiatr. 1999;57(3-A):594-8. 
7. Rotta NT. Acidente vascular cerebral isquêmico em crianças. In: Melo-Souza SE. Tratamento das doenças neurológicas. Rio de Janeiro: Guanabara Koogan; 2000. p. 148-9.

8. Rotta NT. Acidente vascular cerebral hemorrágico em criança. In: Melo-Souza SE. Tratamento das doenças neurológicas. Rio de Janeiro: Guanabara Koogan; 2000. p. 150-1. 9. Jornal da Ciência [homepage na Internet]. Sociedade Brasileira para o Progresso da Ciência [atualizada em 2005 nov 03; acesso em 2010 jan 12]. Acidente vascular cerebral compromete aprendizado das crianças; [aproximadamente 4 telas]. Disponível em:< http://www.comciencia.br/noticias/2005/10/crianca.htm>.

10. Figueiredo NMA. Método e metodologia na pesquisa científica. São Caetano do Sul: Difusão editora; 2004.

11. Brasil. Ministério da Saúde. Comissão Nacional de Ética e Pesquisa. Conselho Nacional de Saúde. Manual Operacional para Comitês de Ética em Pesquisa. Série CNS - Cadernos Técnicos, série A, Normas e Manuais Técnicos, n. 133. Brasília; 2002. 83-91p.

12. Bardin L. Análise de conteúdo. Lisboa: Edições 70; 1977.

13. Matta APC, Galvão KRF, Oliveira BS. Cerebrovascular disorders in childhood: etiology, clinical presentation, and neuroimaging findings in a case series study. Arq neuro-psiquiatr. 2006;64(2-A):181-5.

14. Casa Civil (Brasil). Lei Federal no 8.080, de 19 de setembro de 1990. Dispõe sobre as condições para a promoção, proteção e recuperação da saúde, a organização e o funcionamento dos serviços correspondentes e dá outras providências. Brasília: Diário Oficial da União, 1990 set 19. [acessado em 2009 dez 15]. Disponível em: $<$ http://www.planalto.gov.br/CCIVIL/LEIS/L8080.htm>.

15. Sociedade Brasileira de Doenças Cerebrovasculares. Primeiro consenso brasileiro para trombólise no acidente vascular cerebral isquêmico agudo. Arq neuro-psiquiatr. 2002;60(3A):675-80.

ISSN 1695-6141 